\title{
Septic Loosening of a Total Hip Replacement: Case Report
}

\author{
D.G. Kinyanjui, MBchB, MMed (Nbi), Higher Dip. Ortho (SA), FCS (COSECSA), Department of Orthopedic Surgery, \\ Kenyatta National Hospital, P.O. Box 19983-00202, Nairobi, Kenya
}

\section{SUMMARY}

It can be difficult to differentiate septic from aseptic loosening of prosthesis and especially those due to delayed and late prosthetic-joint infection. In delayed and late prosthetic-joint infection, the acute signs and symptoms of infection such as fever, swelling, erythema and warmth are usually absent and the only manifestation may be implant loosening, persistent joint pain or both; same as aseptic loosening. The patient presented is a 62-year-old farmer who presented with implant loosening and with subtle clinical features that could not help differentiate septic from aseptic loosening. The diagnostic challenges faced and investigations that proved useful to differentiate septic from aseptic loosening are presented.

\section{INTRODUCTION}

Joint replacement is now a commonly performed procedure and implant loosening is a major problem that arthroplasty orthopaedics surgeons have to contend with, as it is one of the most important factors affecting mid-term and long-term results of arthroplasties. Implant loosening can generally be divided into septic and aseptic loosening.

Septic loosening can be due to early (develop less than three months after surgery), delayed or late prosthetic-joint infection (1). Early infection typically presents with acute signs and symptoms such as acute onset joint pain, fever, effusion, erythema and warmth and sometimes with abscess formation with wound breakdown. It is commonly due to virulent organisms such as staphylococcal aureas and gram-negative bacilli and is usually acquired during implantation of prosthesis (2). The diagnosis of prosthetic loosening due to early infection is typically straight forward as clinical features are apparent. Delayed infection is also commonly acquired during implantation of prosthesis but differs from acute infection in that it occurs 3 to 24 months after surgery and acute symptoms of infection are lacking; hence diagnosis may be missed (2). Late infections develop more than 24 months after surgery and are predominantly due to haematogenous seeding. The most common sources of bacteramia are skin, respiratory tract, dental, and urinary tract infections (2).

Microorganisms that grow in biofilms typically cause prosthetic joint infections (3). Within biofilms, microorganisms are enclosed in a polymeric matrix and are protected from antimicrobial agents and host immune responses (4). The microorganisms have also much greater resistance than do planktonic bacteria due to reduced growth rate in the biofilm probably because of incomplete penetration of metabolic substrates, such as glucose or oxygen $(5,6)$.

Aseptic loosening is due to particles, mainly polyethylene wear particles, which induce a macrophage response leading to osteolysis and thus implant loosening (7-10).

\section{CASE REPORT}

S.N was a 62-year-old man who presented for his routine follow up in arthroplasty clinic, Pretoria Academic Hospital, with progressive left hip pain of insidious onset. He had undergone total hip replacement for dysplastic left hip some six years before.

S.N had uneventful postoperative period until about five months before presentation when he started experiencing some mild left hip pain, persistent even at rest but worsened by activities such as walking. However, the pain was not initially serious as to need any analgesics and was not interfering with his routine activities, which included supervising workers in his farm. Over time, the pain progressively worsened necessitating use of nonsteroidal anti-inflammatory drugs and soon started walking with a limp. At presentation he could only walk with crutches and pain persisted throughout the day irrespective of activities. He gave no history of fever, general malaise or weight loss. Examination revealed a patient in pain, was not 
febrile or pale. Vital signs were within normal limits. He was walking with crutches and because of pain, his gait could not be assessed properly. The left lower limb was $3 \mathrm{~cm}$ shorter and was held in internal rotation. There was no hip swelling, erythema or discharging sinus. The left hip was tender to palpation and all hip movements were reduced.

Radiographs showed loosening of both the acetabular and femoral components with displacement of acetabular component posterosuperiorly. There was a periprosthetic fracture involving the lateral cortex of the femur (Figure 1).

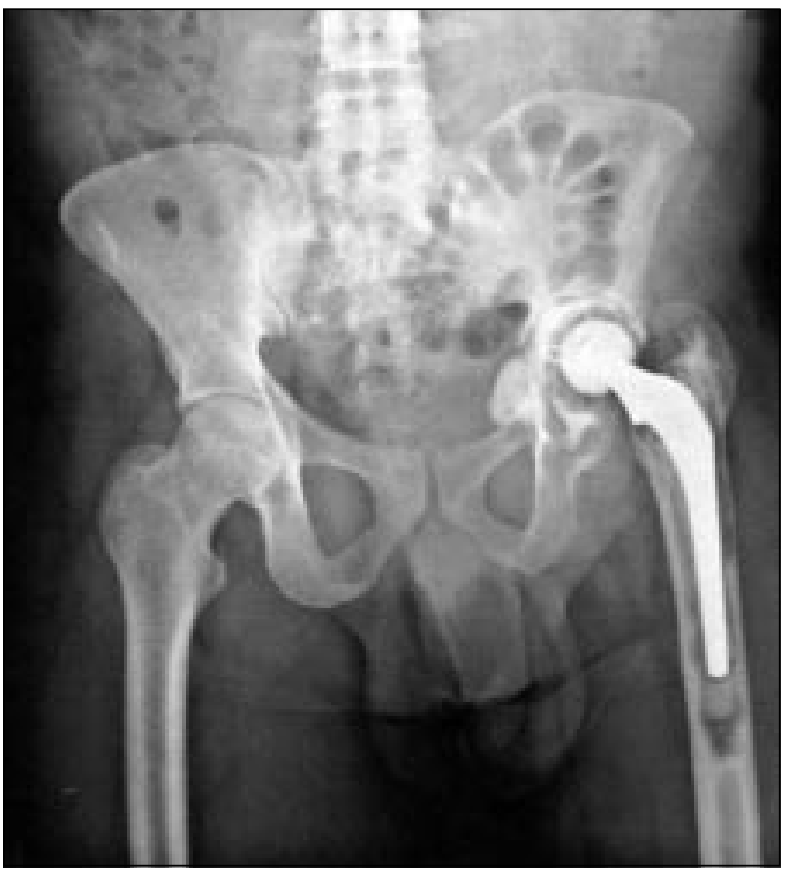

Figure 1: AP view, showing loosening with superior migration of acetabular component and periprosthetic femur fracture.

He had a haemoglobin level of $13.2 \mathrm{~g} / \mathrm{dl}$, WBC of $23 \times 10$,ESR $46 \mathrm{~mm} / \mathrm{hr}$, and C-RP was 115. Urea and electrolytes were normal as well as urinalysis. An impression of septic loosening was made.

The patient was admitted and subsequently taken to theatre for joint aspiration for microscopy, culture and sensitivity. It was a difficult aspiration and only about 1 $\mathrm{ml}$ of bloody fluid could be obtained. Both microscopy and cultures were negative for microorganisms. However, given the history of pain, even at rest, and the laboratory findings, infection could not be ruled out.

The patient was planned and prepared for a 2-stage replacement procedure. He was taken to theatre on $18^{\text {th }}$ May 2006 and removal of implants (both femoral and acetabular) was done. Thorough debridement was done and acetabular and femoral membranes taken for histology, microscopy, culture and sensitivity. After thorough irrigation with normal saline, the hip was closed and drains left in situ. The patient was provisionally started on Kefzol 1g 8-hourly awaiting histology, culture and sensitivity results. He was also on clexane $40 \mathrm{mg}$ daily and analgesics.

Histology showed features of acute inflammation, highly suggestive of infection. Coagulase-negative staphylococcus was grown sensitive to Ciprofloxacin. This lead to the patient being immediately started on ciprofloxacin 750mg 12-hourly and this treatment was to continue for three months. Meanwhile he was mobilized, initially on walking frame and later on crutches. He was discharged on 7th postoperative day, on ciprofloxacin, for follow up in arthroplasty clinic. The clinic follow up was uneventful and he was readmitted six months after the 1st stage procedure, and aspiration for microscopy, culture and sensitivity, full blood count, ESR, and C-RP were done. All were within normal limits.

The patient was prepared for the 2 nd stage procedure which was performed using impaction bone graft to augment the medial, superior and posterior walls, a porous-coated hemisphere shell secured with superior screws, polyethylene insert, ceramic head, and along femoral stem. The lateral wall was augmented with cortical allograft secured with bone cables (Figures 2 and 3). The patient had uneventful immediate postoperative period and is still on follow up in arthroplasty clinic.

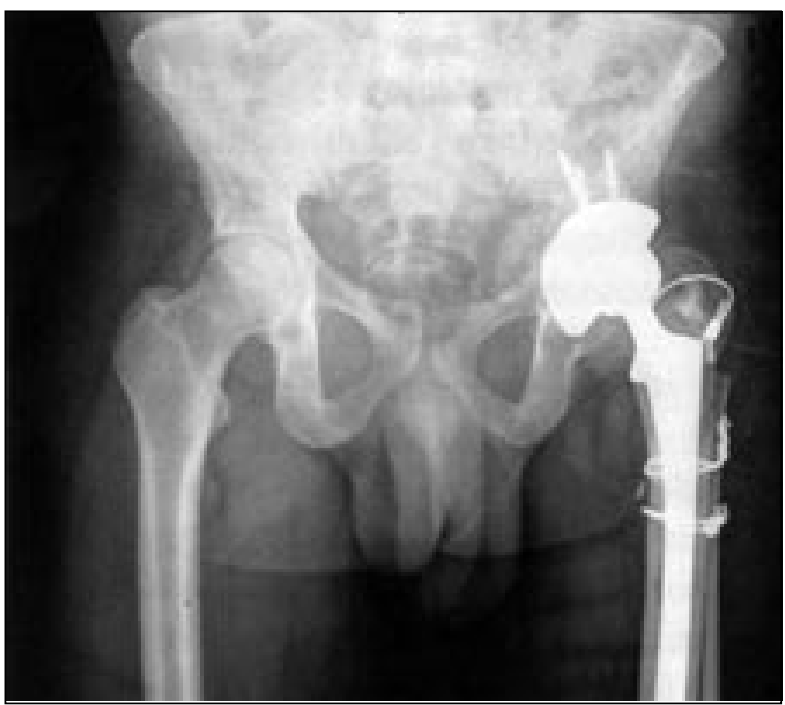

Figure 2: Revision with use of impaction graft, porouscoated shell, and a long femoral stem. The cortical allograft onlay strut is shown, secured with bone cables, to augment the lateral cortex. 


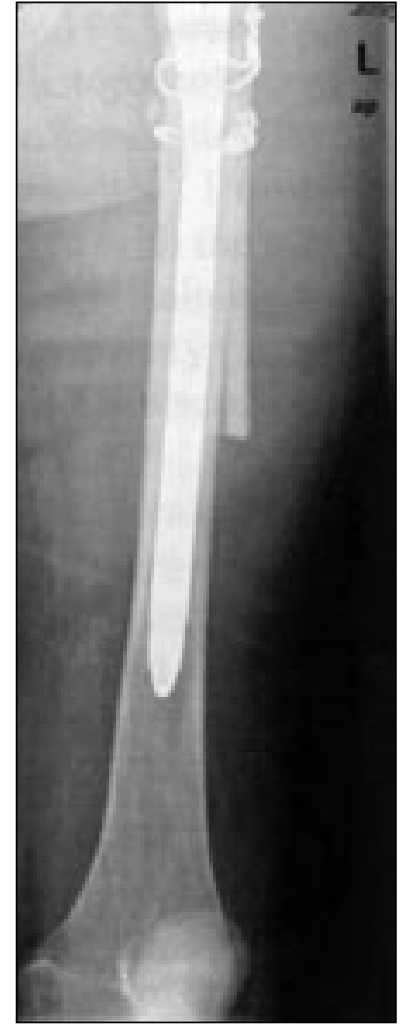

Figure 3: An AP view showing the long femoral stem used.

\section{DISCUSSION}

The above case represents the dilemma that implants loosening may present. Where as there are straightforward cases of septic loosening, there is always some fear of treating delayed and late prosthetic-joint infection as aseptic loosening. Therefore, it is important to differentiate septic from aseptic loosening and, perhaps, safer to assume all implant loosening are septic until proven otherwise.

No uniform criteria have been developed to diagnose prosthetic-joint infection (2).In several studies (11-14), infection was diagnosed if at least one of the following criteria was present: growth of the same microorganism in two or more cultures of synovial fluid or periprosthetic tissue, purulence of synovial fluid or implant site, acute inflammation on histopathological examination of periprosthetic tissue, or presence of a sinus tract communicating with the prosthesis. Commonly cultured organisms are coagulase-negative staphylococcus (30\%-40\%), S. aureas (12\%-23\%), mixed flora (11\%), streptococci (9\%-10\%), gram-negative bacilli (3\%-6\%), enterococci (3\%-7\%) and anaerobes (2\%-4\%) $(15,16)$. No microorganisms are detected in about $11 \%$ of apparent infection.
The above criteria have high specificity but some patients may present with infection that does not meet the above criteria. It is therefore necessary to carry out thorough laboratory and imaging studies in all cases of implant loosening to rule out infection. Leucocytosis and rising C-reactive proteins may be suggestive but not sufficiently discriminative to predict the presence or absence of infection (2). On the other hand a synovial leucocyte count of more than 1700 per cubic millimeter or finding more than $65 \%$ neutrophils has a sensitivity for infection of $94 \%$ and $97 \%$, respectively, and specificities of $88 \%$ and $98 \%$ respectively in patients without underlying inflammatory joint diseases (17). Histopathological examination of periprosthetic tissue showing features of acute inflammation has sensitivity of more than $80 \%$ and a specificity of more than $90 \%$ (18). However, there is a high inter-observer variability and it is important to sample area with the most florid inflammatory changes (2).

Gram staining and microscopy of synovial fluid and periprosthetic tissue has a high specificity (more than 97\%) but generally has low sensitivity (less than 26\%) (18). In aspirated synovial fluid, the pathogens can be detected in $45 \%$ to $100 \%$ of cases (18).

Cultures of periprosthetic tissue provide the most reliable means of detecting the pathogen and are frequently used as the reference standard for diagnosing infections associated with prosthetic joint. Atleast three intraoperative tissue specimens should be sampled for culture $(17,18)$. Swab cultures have a low sensitivity and should be avoided (1). It should be noted that cultures may be negative because of prior antimicrobial exposure, prolonged transport time to the microbiology laboratory, low number of organisms or fastidious organisms $(19,20)$. To detect cases of lowgrade infection, antimicrobial therapy must be stopped two weeks before tissue specimen are obtained (18). Likewise, preoperative prophylaxis should not be given before tissue specimens, if revision surgery is planned (19). Serial plain radiographs may show subperiosteal bone growth and trascortical sinus tract suggestive infection (2). Scintigraphy with technitium-99m has high sensitivity but low specificity where as indium-III -labled autologous leucocytes is time consuming and hence, both are not routinely used $(20,21)$.

The treatment of septic loosening includes one or two stage exchange, resection arthroplasty, arthrodesis or amputation. One-stage revision involves removal of all foreign material, debridement, and reimplantation of a new prosthesis during the same procedure. 
In the above presented case, our going for a two-stage procedure paid off after microorganisms were isolated from periprosthetic tissue biopsies and appropriate antibiotics started based on sensitivity results. It is highly likely that a disaster would have ensued had we managed the loosening as aseptic, based on negative bacteriology of preoperative hip aspiration. Therefore, a combination of clinical, laboratory and radiological assessments are essential before treatment of all suspected aseptic loosening.

\section{REFERENCES}

1. Shafroth M., Zimmerli W., Brunazzi M. and Oschner P. Infections in Oschner P.E., edn. Total hip replacement. Berlin: Springerwelag. 2003; 65-90.

2. Werner Z., Andrej T. and Oshner P.E. Prosthetic-joint infections. New Engl. J. Med. 2004; 351: 1645-1654.

3. Gristinna A.G. Biomaterial-centred infection: Micbiol adhesion versus tissue integration. Science. 1987; 237: 1588-1595.

4. Corsternon J.W., Stewart P.S. and Greenberg E.P. Bacterial biofilm: A common cause of persistent infections. Science. 1999; 284: 1318-1322.

5. Ceri H., Olson M.E., Stremic C., Read R.R., Morck D. and Burret A. The calgary biofilm device: New technology for determination of antibiotics susceptibility of bacterial biofilms. J. Clin. Microbiol. 1999; 37: 1771-1776.

6. Anderl I.N., Zahller J., Roe F. and Stewart P.S. Role of nutrient limitation and stationary-phase existence in Klebsiella Pneumonia biofilm resistant to ampicillin and ciprofloxacin. Antimicrobiol Agents Chemotherapy. 2003; 47: 1251-1256.

7. Willert H.G. and Smith R.L. Reaction of articular capsule to wear products of artificial joint prostheses. J. Biomed. Mater. Res. 1977; 11: 157-164.

8. Maloney W.J., Smith R.I., Schmalzried T.P., Chiba J., Huene D. and Rubash $\mathrm{H}$. Isolation and characterization of wear particles generated in patients who have had failure of a hip arthroplasty without cement. Bone Joint Surg. 1995; 77A: 1301-1310.

9. Kadoya Y., Revell P.A., Al-saffar N., Scott G. and Freeman M.A. Bone formation and bone resorption in failed total hip arthroplasties: histomorphometric technique. Orth. Res. 1996; 14: 473-482.

10. KadoyaY., Revell P.A., Kobayashi A., Al-saffar Mascot G. and Freeman M.A. Wear particulate species and bone loss in failed total joint arthroplasties. Clin. Orthop. 1997; 340: 118-129.

11. Berbari E.F., Hanssen A.D. and Duffy M.C. Risk factors for prosthetic-joint infection: Case-control study. Clin. Infect. Dis. 1998; 27: 1247-1254.

12. Meehan A.M., Osmon D.R., Duffy M.C., Hanssen A.D. and Darkening M.R. Outcome of penicillin-susceptible streptococcal prosthetic joint infection treated with debridement and retention of the prosthesis. Clin. Infect. Dis. 2003; 36: 845-849.

13. Brand C.M., Sistronk W.W. and Duffy M.C. Staphylococcus aureas Prosthetic joint infection treated with debridement and prosthesis retention. Clin. Infect. Dis. 1997; 24: 914919.

14. Tarrevinn P., Cremeinux A.C., Pottier P., Huren D. and Carbon C. Prosthetic joint infection: When can prosthesis salvage be considered? Clin. Infect. Dis. 1999; 29: 292295.

15. Steckelberg J.M. and Osmon D.R. Prosthetic joint infections. In: Waldvogel F.A., Bisno A.L., Edns. Infections associated with indwelling medical devices. $3^{\text {rd }}$ edn. Washington, DC. Amer. Soc. Microbiol. 2000; 173-209.

16. Pandey R., Berendt A.R. and Anthanason N.A. Histological and microbiological findings in non-infected and infected revision arthroplasty tissues. Archorthop. Trauma Surg. 2000; 120: 570-574.

17. Trampauz A., Hanssen A.D., Osmon D.R.., Mandrekar J., Streckelberg J.M. and Patel R. Synovial fluid leucocytes count and differential for the diagnosis of prosthetic knee infection. Amer. J. Med. 2004; 117: 556-562.

18. Trampuz A., Streckelberg J.M., Osmon D.R., Cockreff F.R., Hanssen A.D. and Patel R. Advances in the laboratory diagnosis in prosthetic joint infection. Rev. Med. Microbial. 2003; 14: 1-14.

19. Spangehl M.J., Masri B.A., O'connell J.X. and Duncan C.P. Prospective analysis of preoperative and intraoperative investigations for the diagnosis of infection at the site of two hundred and two revision total hip arthroplasties. J. Bone Joint Surg. 1999; 81: 672-683.

20. Smith S.I., Wastie M.I. and Forster I. Radionuclide bone scintigraphy in the detection of significant complications after total knee Joint replacement. Clin. Radiol. 2001; 56: 221-224.

21. Hain S.E., O'Doheny M.J., Smith. Malfunctioned imaging and the orthopedic surgeon. Bone Joint Surg. Brit. 2002; 84: 315-321. 